\title{
Coulisses
}

Revue de théâtre

\section{Sean O'Casey, La Charrue et les étoiles}

Mise en scène de Irène Bonnaud

\section{David Ball}

\section{OpenEdition}

\section{Journals}

Édition électronique

URL : https://journals.openedition.org/coulisses/954

DOI : 10.4000/coulisses.954

ISSN : 2546-9460

Éditeur

Presses universitaires de Franche-Comté

\section{Édition imprimée}

Date de publication : 31 décembre 2009

Pagination : 19-23

ISBN : 978-2-84867-270-0

ISSN : $1150-594 \mathrm{X}$

\section{Référence électronique}

David Ball, «Sean O'Casey, La Charrue et les étoiles », Coulisses [En ligne], 39 | Automne 2009, mis en ligne le 30 novembre 2016, consulté le 29 décembre 2022. URL : http://journals.openedition.org/ coulisses/954; DOI : https://doi.org/10.4000/coulisses.954

Ce document a été généré automatiquement le 29 décembre 2022.

Tous droits réservés 


\title{
Sean O'Casey, La Charrue et les étoiles
}

\author{
Mise en scène de Irène Bonnaud
}

\author{
David Ball
}

\section{RÉFÉRENCE}

Sean O'Casey, La Charrue et les étoiles. Mise en scène : Irène Bonnaud. Nouveau Théâtre

de Besançon, mars 2009

1 Là où l'Anglais, Harold Pinter, a fait son théâtre des silences et de l'art de la litote et de la dérobade censés être typiques de ses compatriotes, l'Irlandais, Sean O'Casey, a fait le sien d'un autre stéréotype bien porteur, celui de la faconde sentimentale. Et il n'est pas le seul. Nous pourrions dire autant du Synge du Baladin du monde occidental (The Playboy of the Western World) et du Shaw de L'autre île de John Bull (John Bull's Other Island). Quant aux pièces de Wilde ou de Beckett, dont les personnages ne sont pas nommément irlandais, est-il possible d'y entendre l'influence lointaine d'un pays où le bien parler, le bien raconter, sans que la vérité soit toujours scrupuleusement respectée, sont cultivés à tous les niveaux de la société ? On va parfois encore plus loin en posant la même question à propos de Sheridan et Goldsmith au dix-huitième siècle. Dans quelle mesure la réussite de leurs comédies est-elle redevable aux origines irlandaises de leurs auteurs?

2 Aujourd'hui, pourtant, dans une Irlande indépendante, plus prospère et commerçante, multiculturelle, le stéréotype de l'Irlandais beau parleur, grand buveur, peu travailleur, est perçu comme un fardeau du passé dont il faut absolument se débarrasser. De plus, tous les auteurs que nous venons de citer, nous pouvons citer aussi Swift et Sterne, n'étaient irlandais que dans le sens où Camus était algérien, c'est-à-dire qu'ils appartenaient à une minorité dominante venue de la métropole: dans le cas de l'Irlande, des protestants venus de l'Angleterre et de l'Ecosse pour coloniser et administrer une Irlande toujours catholique et qui parlait le gaélique au moins autant que l'anglais. Et comme Camus encore, ils quittèrent tous tôt ou tard le pays natal pour faire carrière en métropole. 
3 Mais une minorité dominante a tout de même ses pauvres, de très pauvres dans le cas de la famille d'o'Casey. Elle a aussi ses rebelles, ses contestataires, ceux qui choisissent le camp des indigènes et le mouvement nationaliste pour l'indépendance, ce qui fut encore le cas d'O'Casey. Né John Casey en 1880, il gaélicisa symboliquement son nom vers l'âge de vingt ans et passa de précieuses heures de son temps libre à apprendre la langue gaélique.

4 La Charrue et les étoiles est la dernière et la plus provocatrice de ses trois grandes pièces irlandaises, toutes consacrées à la lutte armée pour l'indépendance, les deux autres étant L'Ombre d'un franc-tireur et Junon et le paon. Chaque pièce est située à un moment décisif des combats : le soulèvement tragique de Pâques 1916 (La Charrue et les étoiles), la répression britannique de 1920-21 menée par la milice notoire, les «Black and Tans » (L'Ombre d'un franc-tireur), et puis la guerre civile de 1922-23 (Junon et le paon), guerre civile entre, d'un côté, les partisans du compromis négocié avec le gouvernement britannique, c'est-à-dire, l'acceptation que les six comtés du nord de l'Irlande, à majorité protestante, resteraient britanniques, et de l'autre, les nationalistes qui voulaient continuer la lutte pour l'indépendance de tout le pays.

5 Ce sont des pièces réalistes et satiriques, comiques et tragiques à la fois, où la bêtise humaine fait rire avant de faire pleurer. O'Casey y critique et caricature les indépendantistes catholiques, sans doute avec encore plus de férocité et d'amertume qu'il avait lui-même milité à leurs côtés avant de quitter le mouvement en 1914 à cause $\mathrm{du}$ fait que trop peu de nationalistes soutenaient la cause socialiste et ouvrière. O'Casey, au contraire, ouvrier manuel jusqu'à l'âge de quarante-cinq ans, donna toujours la priorité au socialisme et aux luttes ouvrières de sa première inspiration. Dans ses pièces, le discours nationaliste d'une religiosité larmoyante arrive à aveugler les gens modestes aux conséquences inévitables de la violence. Personne ne sait d'avance où vont se terminer les mots de la fanfaronnade, ou les balles des fusils.

6 Au cœur de La Charrue et les étoiles, un jeune couple, les Clitheroe : elle, Nora (jouée ici par Marie Favre), qui aime éperdument son mari et qui veut qu'il reste tranquillement à la maison, et lui, Jack (Assaad Bouab), membre de l'armée clandestine des nationalistes, qui hésite mais qui choisit finalement la lutte armée et trouve la mort lors du soulèvement. Au même moment, Nora perd son enfant mort né et sa raison avec. Sa folie est un écho de celle d'Ophélie à la fin d'Hamlet, un discours décousu de bribes de souvenirs et de chansons qui parlent de fleurs et d'oiseaux. Autour d'eux, les autres habitants de l'immeuble, représenté ici au milieu du plateau par une immense construction à deux étages qui tourne comme un manège avec, d'un côté, la rue, et de l'autre, des intérieurs (décor signé Claire Le Gal).

7 Les gens de l'immeuble, des gens pauvres, obsessionnels, se disputent à cœur joie aux temps normaux, mais aux moments de crise s'entraident, comme Bessie Burgess (Martine Schambacher), loyaliste protestante toujours opposée à ses voisins catholiques, qui aide Nora sans hésitation au moment de son accouchement. C'est en protégeant une Nora folle et, sans idée du danger, se mettant à la fenêtre, qu'elle reçoit la balle d'un soldat britannique qui tire sur tout ce qui ressemble à un tireur embusqué. N'est-ce pas elle, finalement, cette pauvre protestante opposée au soulèvement, qui en est le vrai martyr, la vraie victime sacrificielle, même si - ironie tragique toujours - elle meurt d'une balle tirée de son propre camp ? De toute manière, c'est sur cette mort que la pièce se termine, créant l'impression d'une futilité totale, impression qui fut la cause principale du scandale provoqué par la pièce lors de ses premières représentations, en 
1926, le souvenir du soulèvement de 1916, malgré son échec tout prévisible, étant resté chez les nationalistes irlandais comme celui d'un héroïsme patriotique.

Les personnages féminins d'o'Casey sont presque toujours plus admirables que leurs hommes; la Junon de Junon et le paon en est, sans doute, le meilleur exemple. Ce n'est pas là ce que nous appellerions aujourd'hui du féminisme, plutôt une appréciation du rôle traditionnel de la femme, qui représente et soutient les valeurs de l'amour, de la famille, de la solidarité et du bon sens.

9 Comment, donc, mettre en scène, aujourd'hui, en France, de telles pièces? En respectant à la lettre leur réalisme, leur spécificité historique ? En ce qui concerne $L a$ Charrue et les étoiles, qu'elle parle du soulèvement à Dublin en 1916 et de rien d'autre ? Ou en éliminant la spécificité historique de la pièce, pour qu'elle parle, de manière générale et dans un contexte indéterminé, de la violence politique, révolutionnaire ou nationaliste, et ses conséquences? L'ironie tragique des idéologies contredites par les faits et les événements serait la même dans les deux cas, mais les détails et les références textuelles et contextuelles seraient différents, et peut-être très différents.

10 Pour sa mise en scène, Irène Bonnaud a choisi de faire un compromis entre les deux. $\mathrm{Ce}$ n'est pas en 1916 mais dans un Dublin des années 1980 que se déroulent les événements, ce qui risque de créer plus qu'une absurdité : des soldats britanniques de nos jours qui se battent dans la capitale d'une Irlande indépendante depuis au moins soixante ans! Mais la pièce, malgré tous les détails incongrus, de vocabulaire, de décor, de costume et de musique, reste opiniâtrement au temps de ses origines en 1916. L'excès de mise en scène, cependant, a tendance à diminuer l'impact, tragique et polémique à la fois, de sa structure fort simple : le contraste entre la rhétorique de la mort - qu'il est beau, glorieux, de mourir pour l'Irlande - et la réalité de cette même mort quand, tout bêtement, elle frappe.

11 Mais il faut saluer l'énergie et l'engagement de tous les acteurs, de toute l'équipe, avant de poser, en conclusion, une question plus large : est-il possible aujourd'hui de mettre en scène les pièces classiques mais de style réaliste d'Ibsen ou de Tchekhov (ou d'o'Casey) comme on met en scène parfois les pièces poétiques de Shakespeare et dans une moindre mesure celles de Racine, c'est-à-dire, avec des acteurs de toutes les couleurs et de toutes les origines, dans des décors et des contextes inattendus, avec des rôles d'homme joués par des femmes et vice-versa, en suivant l'argument de base que tous les acteurs devraient avoir la possibilité de jouer dans les grandes pièces du répertoire? Le succès, et l'échec, de cette mise en scène de compromis m'encourageraient à faire une réponse positive.

o'Casey, en quittant l'Irlande, choisit une autre direction. Dans la deuxième moitié de sa carrière, dès la fin des années vingt, il se consacra à un théâtre plus expérimental, plus poétique, plus symboliste ou expressionniste, comme s'il avait entre temps changé de maître, d'Ibsen à Strindberg, un Strindberg néanmoins pour qui le rouge vif n'était pas la couleur éclatante du communisme. Au moment de sa mort, en 1964, c'était peutêtre bien ce deuxième O'Casey qui suscitait plus d'intérêt. Aujourd'hui, pourtant, il me semble que c'est bien le moment de redécouvrir ses trois grandes pièces irlandaises, d'abord pour leur qualité et ensuite parce que l'histoire de l'Irlande, nord et sud, a toujours son actualité, même s'il ne faut demander à une seule pièce de résumer toute l'histoire de l'Irlande au vingtième siècle ! 
La Charrue et les étoiles, photographie de scène

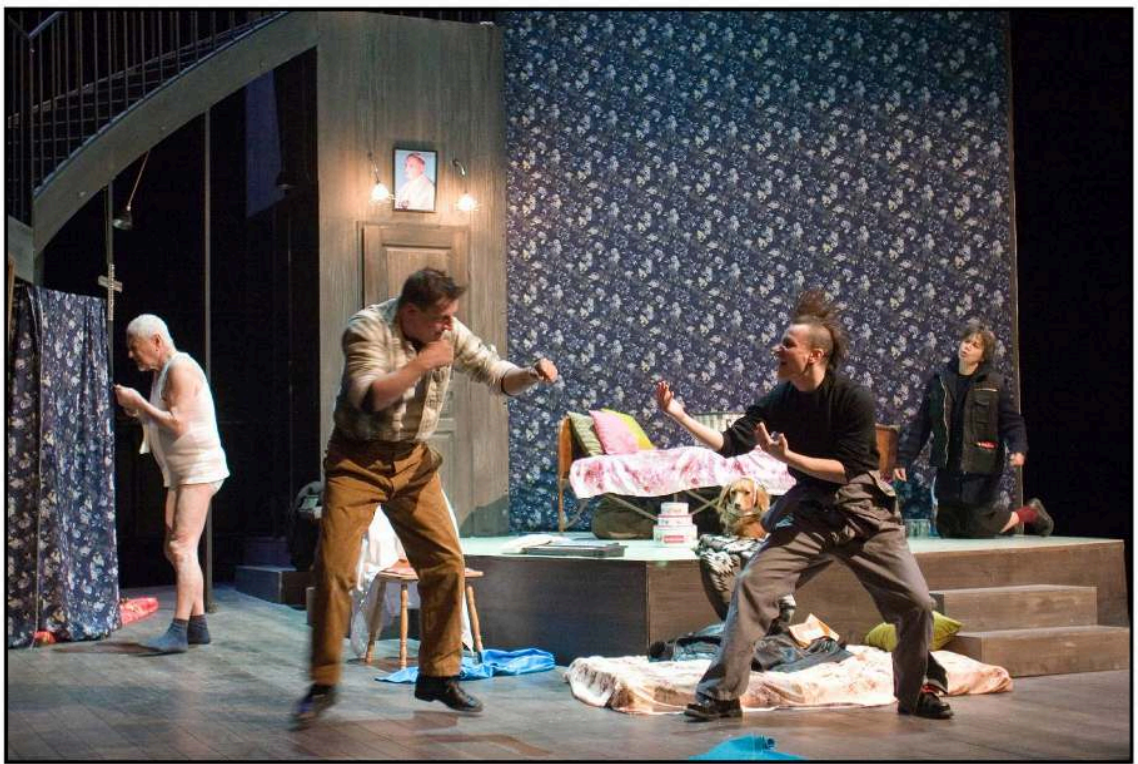

Photo V. Arbelet 